\title{
Exploring Sound Awareness in the Home for People Who are Deaf or Hard of Hearing
}

\author{
Dhruv Jain, Angela Lin, Rose Guttman, Marcus Amalachandran, Aileen Zeng, Leah Findlater, Jon Froehlich \\ University of Washington, Seattle, Washington, United States WA \\ djain@uw.edu, angelacareylin@gmail.com,rguttman@uw.edu, markamal@uw.edu, aileenz@uw.edu, leahkf@uw.edu, jonf@uw.edu
}

\begin{abstract}
The home is filled with a rich diversity of sounds from mundane beeps and whirs to dog barks and children's shouts. In this paper, we examine how deaf and hard of hearing $(\mathrm{DHH})$ people think about and relate to sounds in the home, solicit feedback and reactions to initial domestic sound awareness systems, and explore potential concerns. We present findings from two qualitative studies: in Study 1, $12 \mathrm{DHH}$ participants discussed their perceptions of and experiences with sound in the home and provided feedback on initial sound awareness mockups. Informed by Study 1, we designed three tablet-based sound awareness prototypes, which we evaluated with $10 \mathrm{DHH}$ participants using a Wizard-of-Oz approach. Together, our findings suggest a general interest in smarthome-based sound awareness systems particularly for displaying contextually aware, personalized and glanceable visualizations but key concerns arose related to privacy, activity tracking, cognitive overload, and trust.
\end{abstract}

\section{CCS CONCEPTS}

-Human-centered computing-Empirical studies in accessibility $\cdot$ Human-centered computing-Accessibility technologies

\section{KEYWORDS}

Deaf and hard of hearing, smart home, sound awareness.

\section{ACM Reference format:}

Dhruv Jain, Angela Lin, Rose Guttman, Marcus Amalachandran, Aileen Zeng, Leah Findlater and Jon Froehlich. 2019. Exploring Sound Awareness in the Home for People who are Deaf or Hard of Hearing. In Proceedings of the SIGCHI conference on Human factors in computing systems (CHI 2019). May 4-9, 2019, Glasgow, Scotland, UK, ACM, New York, NY, USA, Paper 94, 13 pages. https://doi.org/10.1145/3290605.3300324

Permission to make digital or hard copies of all or part of this work for personal or classroom use is granted without fee provided that copies are not made or distributed for profit or commercial advantage and that copies bear this notice and the full citation on the first page. Copyrights for components of this work owned by others than ACM must be honored. Abstracting with credit is permitted. To copy otherwise, or republish, to post on servers or to redistribute to lists, requires prior specific permission and/or a fee. Request permissions from Permissions@acm.org.

CHI 2019, May 4-9, 2019, Glasgow, Scotland UK

(C) 2019 ACM. ACM ISBN 978-1-4503-5970-2/19/05 \$15.00

https://doi.org/10.1145/3290605.3300324

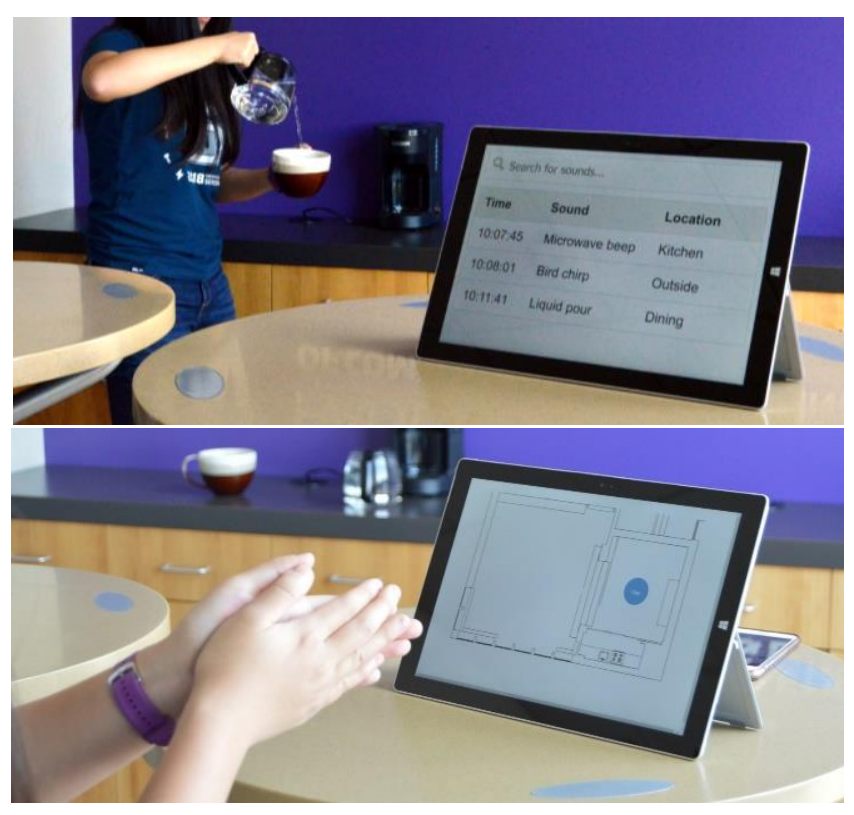

Figure 1: Two of the three sound awareness prototypes we used for Wizard-of-Oz evaluations: (a) list and (b) floorplan. Actions performed: (a) liquid pouring, (b) a clap.

\section{INTRODUCTION}

The home is filled with a rich diversity of sounds that convey information about the home environment and the occupants within it-from mundane beeps and whirs to children's shouts and dog barks. For deaf and hard of hearing (DHH) people, designing and adapting a home space can mean arranging furnishings, mirrors or lighting to allow for clear sightlines and visual awareness [24], and installing visual and vibrational options for urgent information that is typically conveyed to hearing people via sound (e.g., alarm clock, doorbell).

At the same time, many DHH individuals are interested in having greater access to sound awareness in the home $[2,14]$. In 2006, before the recent proliferation of smarthome technologies, Matthews et al. [14] interviewed $18 \mathrm{DHH}$ participants in two studies about sound awareness needs at home, at work, and while mobile, finding that at home, participants were most interested in emergency alarms, people shouting, and appliance sounds. 
More recently, Bragg et al. [2] conducted an online survey with $87 \mathrm{DHH}$ participants, confirming that emergency alarms, appliance information, and door knocks/doorbell were among the most desired sounds. Further, about half of both deaf and hard of hearing participants in the latter study reported missing a sound of interest on a daily basis.

In this paper, we extend and confirm prior work $[2,12,18]$ by providing a more comprehensive and targeted investigation of sound awareness needs, concerns, and solutions in domestic spaces. We also introduce initial sound awareness prototypes designed specifically for the home-as opposed to an office environment [2,14] or for mobile or wearable use (e.g., $[16,17]$ )-evaluate the prototypes in a home-like space, and identify themes that are particularly relevant to adoption in a home context (e.g., issues with activity tracking, privacy).

To explore experiences with sound in the home and to solicit feedback on initial sound awareness mockups, we conducted semi-structured interviews with $12 \mathrm{DHH}$ participants (Study 1). Preferences for sounds of interest, sound characteristics (e.g., location, loudness), and the devices on which to display this information (e.g., a wallmounted device) echo previous findings [2,14]. We also uncovered new themes, such as the need to show different information for different types of sounds and to select sounds based on the user's location in the home, as well as issues of privacy, cognitive overload, and uncertainty of sound classification.

Informed by Study 1 and past work [14], we designed three initial sound awareness prototypes (Figure 3) and conducted a Wizard-of-Oz study with $10 \mathrm{DHH}$ participants (Study 2). This study elicited reactions to the prototypes using short real-time demos and thematic scenarios designed around issues of privacy, cognitive overload, and activity tracking. We uncovered several novel design suggestions to better support domestic life, including contextual factors to control the information shown (e.g., amount of activity in the home), ways to increase the actionability of the system (e.g., showing both start and end times for continuous sounds such as water running), and different visual features to accommodate inaccuracy (e.g., showing source location).

Our research contributes: (1) qualitative results from two studies highlighting needs and challenges related to domestic sound for DHH occupants; (2) early prototype designs for sound awareness in the home; and (3) design recommendations for future in-home sound awareness technologies. While smarthome technology has long been a topic of interest within HCI and Ubicomp, its recent mainstreaming via products like Amazon Echo and Google Home presents new opportunities and challenges for users with disabilities (e.g., [19]). Through a human-centered design process, this paper presents a forward look into how smarthome systems may benefit homes with one or more $\mathrm{DHH}$ occupants and also uncovers and enumerates key concerns. Our findings have implications not just for the accessibility community (i.e., those working on sound awareness solutions for $\mathrm{DHH}$ people) but, more generally, for designers and researchers working on smarthome technology as well.

\section{RELATED WORK}

We survey prior work related to sound awareness needs, tools to provide sound awareness, and smart home systems.

\section{Understanding Sound Awareness Needs}

Several studies have examined general sounds of interest for $\mathrm{DHH}$ people $[2,14,16,20]$, though only a few have specifically included questions about the home [2,14]. As mentioned earlier, Matthews et al. [14] and Bragg et al. [2] identified several sounds of interest in the home, which helps motivate our work.

Although not focusing specifically on sounds in the home, other studies have identified sounds of interest that could apply to the home. Sicong et al. [20] surveyed 60 DHH participants aged 10-26 for their preferred sounds; social sounds (e.g., presence of people, a door knock) and what they termed "early warning events" (e.g., microwave beep, fire alarm) were of high interest. Mielke et al. [16] interviewed six $\mathrm{DHH}$ people, identifying smoke/fire alarms, phone ringing, siren, and doorbell as the most preferred sounds.

Our work builds on the above studies by exploring domestic sounds of interest in more depth, including how preferences may vary in ways that are unique to the home, such as room layout and types of rooms and occupants.

\section{Sound Awareness Tools for DHH People}

Hearing aids and cochlear implants are essential to improving sound awareness for DHH users. Nevertheless, several studies [12,15] report low usage satisfaction with aids due to problems with background noise, fit and comfort issues, and high cost. Commercial products-such as flashing doorbells and vibrating wake-up alarmsprovide haptic or visual alternatives to some information typically conveyed by sound. While useful for their specific applications, these devices do not offer a general alternative to sounds of interest in the home.

In the research literature, Matthews et al. [14] evaluated a desktop sound awareness system with four $\mathrm{DHH}$ people in an office setting, identifying preferred 
sound information (e.g., specific sound, source location) and visualizations for showing this information (e.g., a spectrograph). In formative interviews with eight $\mathrm{DHH}$ participants reported in the same paper, participants felt that a large wall display would be preferred to a PDA or desktop display for home-based sound awareness, although participants did not use such a display. This work was conducted 12-15 years ago; our exploration includes a wall display but also more modern display options (smartphone, smartwatch, HMD). More recently, Sicong et al. [20] and Bragg et al. [2] used smartphones to recognize and display environmental sounds (e.g., phone ringing, sirens), conducting evaluations with 86 and 12 DHH participants respectively. Participants in both studies wanted both visual and vibration modalities to notify about sounds and a custom notification style for each specific sound (e.g., using a different vibratory pattern).

Finally, wearable solutions for sound awareness have also emerged. For example, Jain et al. [11] used a design probe method to explore sound visualizations on a headmounted display with $24 \mathrm{DHH}$ participants. Mielke et al. [14] conducted a Wizard of $\mathrm{Oz}$ exploration of a smartwatch-based app with six DHH participants. The latter work, although preliminary, suggests that participants valued the smartwatch form factor because it is not easily recognized as an assistive technology and has a private display.

In contrast to these studies, our designs focus on sound awareness in the home, and we include a Wizard-of-Oz evaluation of tablet-based sound awareness prototypes.

\section{Smart Home Systems}

HCI studies of technology in the home dates back to the early 1990s. Technology that best improves quality of life integrates into the domestic processes of household members [6,21,22], adapts to user feedback [18,23], uses environmental context to control displayed information $[6,10]$, and hides private information [5,10]. For people with disabilities, smart home systems offer additional benefits [7,19]-for example, Pradhan et al. [19] found that smart speakers, in some cases connected to smart home devices, can increase independence for users with visual and motor impairments. For DHH users, the recent introduction of tablet-based smart home technology (e.g., [25]) offers accessible alternatives to existing voicecontrolled systems, which have trouble understanding deaf speech [1,9]. Our work is complementary and could ultimately be incorporated into a broader smart home ecosystem.

\section{STUDY 1: FORMATIVE AND DESIGN PROBE}

To assess the needs and potential for sound awareness systems in the home, we conducted a two-part study with $12 \mathrm{DHH}$ participants: a semi-structured interview followed by design probes. We first outline a design space that informed the study, before describing the method and findings.

\section{Design Space for Home Sound Awareness}

We generated six design dimensions for a home-based sound awareness system based on prior work in domestic computing $[6,10]$ and technology for DHH users $[2,14]$ :

1. Form factor: What device is used to convey sound information (e.g., smartphone, wall-mounted display)?

2. Output modality: Via what sensory mode does the user receive information (e.g., visual, vibrations)?

3. Display elements: What information about sound is conveyed (e.g., sound type, location, length)?

4. Sound type specificity: How precisely is the sound type conveyed, from the very specific (e.g., "fan on low mode") to moderately specific (e.g., "fan running") to more general (e.g., "whirring sound")?

5. Sound location specificity: How precisely is the sound location conveyed, from very specific (e.g., "upstairs bathroom sink") to more general (e.g., "upstairs").

6. Confidence level: How to convey level of certainty of sound classification (e.g., percentage accuracy)?

\section{Method}

\section{Participants}

We recruited $12 \mathrm{DHH}$ participants (seven females and five males) through email, social media, and snowball sampling. The number of participants was determined based on reaching thematic saturation (see Data Analysis). Participants were on average 36.8 years old $(\mathrm{SD}=16.3$, range $=21-67$ ). Eight participants reported onset of hearing loss as congenital, two reported 1 year, one reported 2.5 years, and one reported 6 years. Nine participants used digital hearing aids and one used cochlear implants. As our study focuses on the home, we also asked about living arrangements: number of rooms, number of total occupants, and number of $\mathrm{DHH}$ occupants. 
These details and others, including cultural identities and reported hearing loss level, are shown in Table 1 . Note that no household had children. Finally, P2, who identified as deaf-blind, reported 20:20 acuity with glasses and peripheral vision less than 20 degrees. He was able to fully engage with our protocol and reported no problems in seeing our visual designs.

\section{Procedure}

The study procedure, conducted by the hard of hearing first author, took about 50 minutes. In addition to verbally questioning participants, the discussions were supplemented visually through illustrations, examples, and questions on an iPad. A real-time transcriptionist attended all study sessions, and participants were given the option of having a sign language interpreter; six participants opted for this accommodation. The study began with a demographic and background questionnaire, followed by a two-part protocol:

Part 1: semi-structured interview. We asked about needs, challenges, and current strategies to access or mitigate the need to know about sounds in the home, as well as ideas for new technologies to address these challenges.

Part 2: design probe. For each dimension of the design space, we provided a brief textual description that included examples of design options (e.g., different display elements or levels of sound type specificity). For the form factor and output modality dimensions, we also presented in random order the five low-fidelity illustrations shown in Figure 2A to 2E, along with the haptic feedback example shown in Figure 2F. Participants chose one or more examples as their preference for a given dimension, and could describe and/or draw a new possibility. We asked for rationale on the choices as well as follow-up questions (e.g., "Would your choice change for different sounds?”). All dimensions were presented in the order listed in the design space above; however, toward the end, we discussed form factors a second time to see if preference had evolved. Finally, because sound sensing is inherently uncertain, we asked whether and how participants would want to see the system's confidence in the sensed sound.

\section{Data Analysis}

We applied an iterative thematic coding approach [3] to the session transcripts. Upon receipt of the first six transcripts, one researcher randomly selected two transcripts and developed an initial codebook for each interview section (i.e., challenges, current strategies, future technology ideas, and the six design space dimensions) and identified a small set of emergent codes that applied holistically across all questions (e.g., privacy, information

\begin{tabular}{|c|c|c|c|c|c|c|c|}
\hline ID & Age & Gender & Identity & Hearing Loss & \#Rooms & \#Occ. & \#DHH \\
\hline P1 & 24 & $\mathrm{~F}$ & $\mathrm{HH}$ & Profound & $10+$ & 7 & 1 \\
\hline P2, R3 & 60 & M & deaf-blind & Severe & $1-3$ & 1 & 1 \\
\hline P3, R4 & 55 & M & Deaf & Severe & $10+$ & 2 & 1 \\
\hline P4, R1 & 54 & $\mathrm{~F}$ & Deaf & Profound & $4-6$ & 1 & 1 \\
\hline P5, R7 & 67 & M & Deaf & Severe & 7-9 & 2 & 1 \\
\hline P6 & 25 & $\mathrm{~F}$ & deaf & Profound & $1-3$ & 1 & 3 \\
\hline P7 & 21 & M & Deaf & Profound & $4-6$ & 1 & 4 \\
\hline P8 & 25 & $\mathrm{~F}$ & deaf & Profound & $1-3$ & 1 & 2 \\
\hline P9 & 25 & M & Deaf & Severe & $4-6$ & 1 & 2 \\
\hline P10, R9 & 33 & $\mathrm{~F}$ & Deaf & Profound & $4-6$ & 1 & 1 \\
\hline P11 & 32 & $\mathrm{~F}$ & Deaf & Profound & $7-9$ & 1 & 3 \\
\hline P12, R6 & 21 & $\mathrm{~F}$ & $\mathrm{HH}$ & Mild & $10+$ & 4 & 2 \\
\hline R2 & 45 & M & deaf & Severe & $1-3$ & 1 & 1 \\
\hline R5 & 54 & $\mathrm{~F}$ & $\mathrm{HH}$ & Severe & 7-9 & 3 & 1 \\
\hline $\mathrm{R} 8$ & 21 & M & $\mathrm{HH}$ & Moderate & $4-6$ & 1 & 1 \\
\hline R10 & 21 & $\mathrm{~F}$ & $\mathrm{HH}$ & Profound & $1-3$ & 1 & 1 \\
\hline
\end{tabular}

Table 1: Participants in Study 1 (P1, etc.) and Study 2 (R1, etc.). Counts for total occupants (\#Occ.) and for DHH occupants specifically (\#DHH) include the participant.

overload). The researcher then coded the remaining four transcripts, updating the codebook as necessary. After these first six, transcripts were coded one by one until we reached thematic saturation, which occurred at 12 participants. The researcher then performed another pass on all transcripts. The final codebook contained 7-9 codes for each interview section. Finally, two other researchers split the set of transcripts to review all codes, agreeing with the first researcher on $96.5 \%$ of the code assignments. The three researchers resolved disagreements through discussion.

\section{Findings}

We discuss sounds of interest, existing adaptations and preferences for in-home sound awareness technology.

\section{Part 1: Formative Interview}

Sounds of interest: Similar to past work [2,8,13], the sounds of greatest interest were alarms and alerts $(N=12)$, appliance timers (10), presence of other people and animals (9), and voices directed at you (7). In contrast, most participants (10) did not want to be aware of continuous background or mechanical noises unless the sound indicates a mechanical problem (e.g., a water leak) or emergency (e.g., a siren) (also identified in [2]). Finally, although participants were interested in sounds indicating presence of people, five participants did not want repeated notifications of that activity, such as creaks, furniture movement, and walking back and forth.

Existing adaptations and challenges: To identify sounds in the home, participants used traditional approaches such as asking for help from other people (5), moving around the house to find the source of perceptible sounds they could not identify (3), and using dogs as guides (3). Several participants also used visual or 


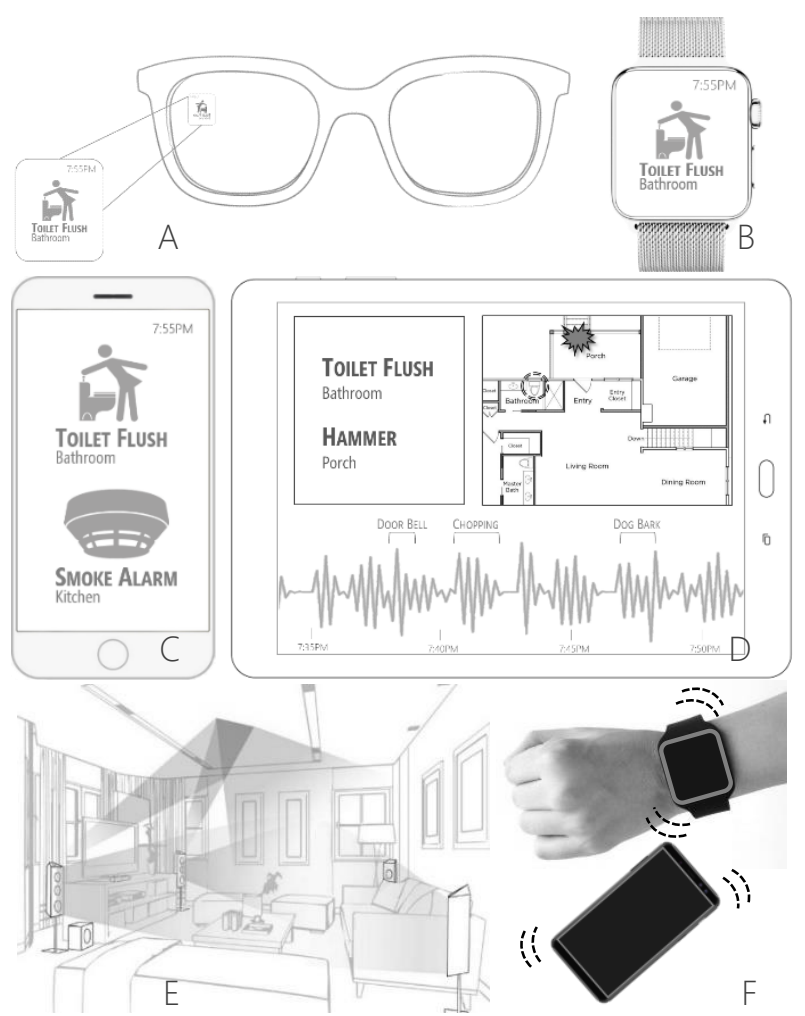

Figure 2: Mockups shown to Study 1 participants to guide them in considering form factors (A-E) and output modality (visual A-E and vibration $F$ ) dimensions. The form factors are: A: HMD, B: smartwatch, C: smartphone, D: wall-mounted, E: ambient display. The ambient display shines light to illuminate sound sources within a room.

vibrational alternatives to what are typically auditory devices: doorbells that flash (7) or vibrate the bed (2), a vibratory alarm clock (6), and a wall-mounted light to display the ambient sound level (3).

When asked about sounds for which they do not have sufficient adaptations, participants mentioned voices (8) and activity sounds (7). In addition, some participants did not have techniques to deal with mechanical sounds (5), outdoor sounds (5) or animal sounds (4), but these sound types, as mentioned by four participants, were "more of nice to haves instead of need to knows" (P4). Notably, six of the seven participants who used one of the standalone sound awareness devices described earlier (e.g., flashing doorbell) deemed the devices to be insufficient. For example, four participants said they do not like flashing doorbells because the light can only be seen from the room in which it is installed, or the system can trigger falsely. An additional three participants emphasized the hassle of installing multiple devices, such as:

"I have [a] flashing doorbell... But, one day I was sleeping and somebody came at night [and] rang the doorbell, and I couldn't see the light. So, I had to get a bed shaker [for the doorbell...] How many devices should [I] keep?"(P11)

Ideas for future technology: When asked to envision a future technology that provides sound awareness in the home, participants' descriptions most commonly mentioned smartphones (9) followed by wall-mounted or tabletop displays (5). Other ideas included smartwatches, head-mounted displays, subtle notifications on everyday devices (TV, computer), and a ring-mounted device because, "the phone doesn't help as overnight it is charging in kitchen, but I never take off my ring"(P5).

Part 2: Design Probe

Form factors: Participants discussed the perceived advantages and disadvantages of the five form factors introduced in the design probe (Figure 2). Only three participants liked the HMD, which, among other criticisms, was seen as visually intrusive. We summarize responses to the other form factors in decreasing order of preference.

Smartphone: All participants liked the smartphone because it is portable (6), a device they already own (6), and close at hand (5). Five participants also valued being able to remotely monitor sounds in the home while they are away.

Wall-mounted: Nine participants liked the wallmounted display, citing benefits of size (compared to smartwatches and smartphones) (6), and static placement in the home (4). However, five participants noted that a downside of the wall display is that it needs to be within their line of sight.

Smartwatch: Eight participants liked the smartwatch, primarily because it is always situated on the wrist (and could be useful for notifications of urgent sounds), and because of its portability. At the same time, the smartwatch was less preferred than the smartphone because most participants do not already wear one (10) and it has a smaller screen (3).

Ambient: Six participants liked the ambient display, where objects making sounds within a room would be visually lit up. Advantages cited by these participants included that the ambient display would offer good visibility and would convey specific sound sources well. For example,

"[Unlike a smartphone], you don't have to try and figure out where the sound is coming from. You can tell immediately. Like if you are here in the room, you will see the light from the [source] and you will know where the sound is coming from and whether you should pay attention to it." (P1)

However, other participants (4) worried about cost and installation effort for the ambient display as well as visual 
clutter, and so only wanted to use this form factor for some specific sounds (e.g., alarms and doorbells).

Output modality: All participants wanted visual information for all types of sounds, but 11 of the 12 also desired vibration for when immediate attention is needed or while sleeping. Six participants felt that vibration to convey the presence of sound paired with a visual display for more information would be useful. We also asked about olfactory feedback, which five participants were receptive to, mainly for emergencies and alerts (5). For example, P12 said: "If I' $m$ in a different room and there's a smoke alarm sounding, [...] a powerful blast of scent could grab my attention.” However, four participants did not want to use smell.

Display elements: We asked about six potential display elements: sound type, location, temporal history, length of occurrence, physical characteristics (e.g., loudness), and importance. All participants wanted sound type and location, reflecting past work [14], while at least eight participants wanted each of the other elements. However, eight participants also felt that extra details would be unnecessary for sounds occurring in the same room as the user. When asked about the utility of these elements for different types of sounds, sound type and location were seen as sufficient by many participants (7) for sounds requiring immediate attention, namely, alarms, alerts, and voices. The additional information could be useful, however, to provide context for activity sounds (7), such as the length of a sound being related to urgency:

"Like for footsteps: how long are they heard? If my housemate is pacing back and forth, it could be something wrong. That could provide me an opportunity to check if everything is alright." (P3)

Sound type specificity: No clear pattern emerged, indicating a need for a more grounded evaluation, as supported by P11: "This is one of those things where I feel I would need to try a system on my own and try filtering it and testing it."We revisit this design dimension in Study 2.

Sound location specificity: Most participants (8) wanted locations to be at least moderately specific (i.e., to display the room in which the sound occurred), for example: "I have a big house [...] if somebody is knocking, which door, front door or back..." (P3)

The remaining participants felt that the need for location specificity would depend on the importance of sound: all wanted a more specific location for more important sounds.

Confidence level: Participants were evenly split on whether to display the system's confidence level of the sensed sound. For example, P8 wanted the confidence level: "because I am curious [on] how well the technology is going to work. And how I can improve [the system] to detect better in future," whereas P1 did not need the confidence level because she: "would only use the technology if it's accurate [in detection]." As with sound type specificity, a more grounded evaluation may be useful.

Other considerations: Several emergent themes pointed to issues unique to a sound awareness device in the home. For example, P7, who lived with three other people, felt that his preferences for sound awareness "would be different if I lived in a house by myself." Privacy was also mentioned by six participants, which included interpersonal privacy concerns. P1, who lived with six hearing adults, said: "I don't want wall mounted in other people's rooms [in the house]. Only maybe in my bedroom and bathroom because I only need to know this information." Finally, comments about information overload, a concern for eight participants, included that continuous, familiar sounds in the home may not need to be displayed:

"If it's going to remind me every five minutes that tools are making noise in the workshop and that there's a motor sound in the bedroom, that could be very annoying" (P6).

\section{Summary}

Participants appreciated the idea of sound awareness in the home, and suggested showing alarm and alerts, presence of other people and animals, activity sounds and voices directed at them. Smartphone and wall displays, and a combination of visual and vibration modalities were the most preferred. Participants suggested customizing what sound information to show (e.g., location, length of occurrence) based on the type of sound. Finally, themes of privacy, multiple occupants, and information overload emerged, which we explore further in Study 2.

\section{STUDY 2: WIZARD OF OZ EVALUATION}

To gain further insight into in-home sound awareness technology, we designed and performed a Wizard-of-Oz evaluation of three sound awareness prototypes in a home-like space (see Figure $3 \mathrm{~b}$ for layout). These prototypes enabled further investigation into themes that emerged in Study 1 but are difficult to study through interviews and static design probes.

\section{Wizard of Oz Prototypes}

Informed by Study 1 and past work [14], we created three web-based prototypes that employ different approaches to displaying sounds and that included elements meant to elicit discussion on themes such as privacy and location. The prototypes, also shown in Figure 3, were:

List: This prototype displays sound activity using a scrolling list. New sounds appear at the bottom of the list 


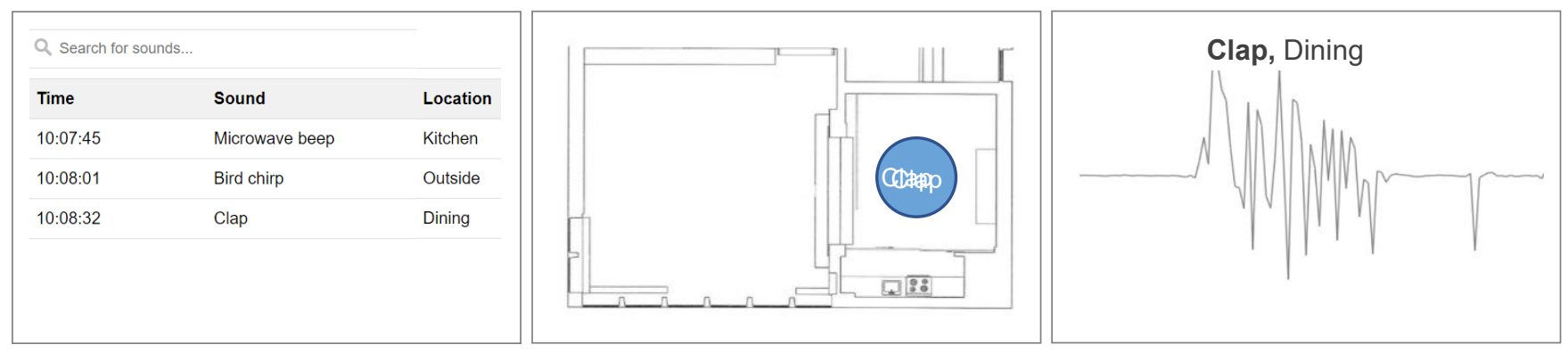

Figure 3: The three prototypes used for Study 2 Wizard-of-Oz evaluation sessions: list, floorplan, and waveform.

with the time of occurrence and the room location. A search bar at the top allows the user to search for specific sounds, and thus access history.

Floorplan: This prototype shows the spatial layout of the house. Sensed sounds appear as fixed-sized blue bubbles for three seconds within the room where the sound occurred. This instantiation demonstrates moderate location specificity, at the room level, but also allowed us to discuss other levels of specificity (house, specific object in room). Sounds from outside of the house appeared to the right of the spatial layout.

Waveform: This prototype includes a continuous scrolling waveform of sound level (loudness) based on a single microphone as input, with text describing the recognized sound and location above the waveform. This design allowed us to investigate the perceived utility of physical sound information, as well as the possibility of only showing a waveform, which is relatively easy to implement, rather than more advanced sound identification. While perhaps less approachable than the other designs, as users gain experience they may be able to extract information from the waveform's depiction of physical sound.

The Wizard of $\mathrm{Oz}$ setup consisted of a Microsoft Surface Pro 3 tablet, which displayed the prototypes in a web browser, and an HTML-based wizard interface that ran on a separate laptop and communicated with the tablet over wifi via a web socket connection. The wizard interface included location buttons the four rooms at the study setting (dining, kitchen, bathroom, lounge, outside) and a list of pre-set sounds that were likely to occur during the study, along with a textbox to enter other sounds. Additionally, for the waveform prototype, we connected a conference microphone to the tablet and used Mozilla's Audio API to generate a real-time waveform.

\section{Method}

\section{Participants}

We recruited $10 \mathrm{DHH}$ participants (five females; five males) through email lists and snowball sampling (Table
1). Because this study extended Study 1, we did not exclude repeat participants. As a result, six Study 1 participants also volunteered for Study 2. Participants were on average 44.1 years old ( $S D=15.7$, range 21-67). Seven reported onset of hearing loss as congenital, and the other three reported 1, 6 and 15 years. All participants used digital hearing aids. Most (7) reported living in a home or an apartment with at least 4-6 rooms. Four participants lived with at least one adult; one of the occupant's in R5's home was a child.

\section{Procedure}

Study sessions were held in a student and faculty lounge on a university campus. This setting was chosen to offer control across participants but also to be home-like: it contained a kitchen, dining room, lounge area (like a living room), bathroom, and windows to the outside. The protocol was designed to take an hour, including a written background questionnaire and three longer activities: initial prototype demos, thematic scenarios, and a semistructured interview. Similar to Study 1, the protocol was conducted verbally with pre-planned questions also presented on an iPad. A real-time transcriptionist attended all sessions. Participants were additionally offered sign language interpretation if desired, which two participants ultimately requested. Three research team members were present during the sessions: the lead facilitator, a wizard, and an actor. The participant, facilitator, transcriptionist, and interpreter (if present) sat at the dining table, while the wizard sat on a couch in the lounge area behind the participant and the actor moved around the area as needed.

Part 1: initial demos and design ideation (10 mins): The three prototypes were demonstrated in random order. These Wizard of $\mathrm{Oz}$ demonstrations were designed to briefly introduce participants to the features of each prototype and to give a sense for how the prototype worked for a small set of everyday domestic tasks. The actor performed three sets of everyday actions: (1) Starts the microwave then does dishes in the kitchen [microwave 
starts, beeps after 10 seconds, water running, dishes clinking]. (2) Knocks at the door [door knocking], which is opened by the facilitator [door open]; after greetings [speech (greetings), door close], sits next to the participant. (3) Makes coffee [machine starts, stops, liquid pouring, object placed], while bird chips outside [bird chip]. For each participant, these sets were randomly paired with the three prototypes. Participants were also encouraged to make their own sounds (e.g., clap, table tap). After the demos, participants explained their reactions to each prototype, suggested improvements (if any), rated the usability of the prototypes, and were invited to sketch new design ideas on blank templates of the prototypes.

Part 2: thematic scenarios (20 mins): Following the initial demos, three new scenarios were presented, one for each prototype. These scenarios were designed to explore themes that could manifest in unique ways in the home setting: privacy, activity tracking, information overload, use of spatial layout, and sounds of interest. Each scenario was first described as follows (without the bracketed sounds), then for the bathroom and movie scenario, the actor played out the scenario as the prototype displayed the sounds:

Bathroom Scenario: You are in your dining room with a fa mily member. You are reading a book. The family member gets up and goes to the bathroom [footsteps, door open/close] while you continue reading. [bathroom sounds (e.g., toilet flush, water running)] The person comes back but forgot to fully close the sink faucet [water dripping].

Babysitter Scenario: You come home from work at 5PM. While you were gone, you left your kids with a babysitter. You're interested in knowing the activities in your house that occurred since your baby woke up from its nap at 4PM. You go and check the history of sounds. [Example sounds include baby crying, baby laughing, toys rattling, liquid pouring, singing].

Movie Scenario Imagine you are in your dining room working on your laptop [keyboard typing]. It is a hot summer evening, so you have your AC on [AC hum]. Your roommate is in the lounge watching television while on the phone with their partner [Sounds from TV (e.g., music, children playing, street sound) as well as phone ringing, roommates' speech, furniture sounds].

We paired each scenario with a default prototype that would better generate reactions to the specific themes we wanted to explore in that scenario. For example, pairing the babysitter scenario with the list prototype (that shows history) allowed us to study the theme of "activity tracking”. Similarly, the bathroom scenario was paired by default with the floorplan prototype, and movie with the waveform prototype. However, all three prototypes were open in the browser for each scenario and we encouraged participants to explore them. For the bathroom scenario, the actor waited outside the study location and the wizard produced sounds as if the actor were using the bathroom. For the babysitter scenario, 18 pre-selected sounds were shown (examples above). We elicited participants' initial reactions after each scenario.

Part 3: semi-structured interview (30 mins): Finally, we asked semi-structured questions about the participants' experience with the prototypes, focusing on information overload, privacy, and activity tracking. We also showed three additional simple mockups for conveying system uncertainty in sound sensing, to further examine this theme from Study 1: showing only the location of a sound within a room but not the type of sound (from [14]), showing a list of possible sounds with accuracy, and showing only a waveform of sound loudness.

\section{Data Analysis}

We analyzed the professional transcripts using a process similar to Study 1. One researcher selected two transcripts to develop an initial codebook, then iteratively applied the codebook to all transcripts (while refining the codebook). The final codebook contained 6-8 codes for each of the five sections-three sections of the transcripts and two additional themes (personalization, installation) that emerged across the entire transcripts. A second researcher then conducted a peer review of all coded data, agreeing in $96.0 \%$ of cases with the initial researcher. Disagreements were resolved through consensus.

\section{Findings}

All participants reacted positively to the idea of sound awareness feedback in the home. For example, R4 on seeing a 'bird chirp' visualization said: "I can only hear birds in the forest alone when nobody is around and I turn my hearing aids all the way up. Cool!" Similarly, R5 said:

"I get real anxiety when my husband is traveling for work. Our bedroom is on the first floor and [our] daughter's room is downstairs. I wouldn't hear if she needs my attention. To have something like this in my home would be amazing." (R5)

However, participants also highlighted challenges with using a sound awareness system in the home. We discuss reactions to our prototypes and insights on the high-level usability themes that arose (e.g., privacy, trust).

\section{Sound Awareness Prototypes}

When discussing their preferred designs, nine participants selected the floorplan because it intuitively visualized the position and type of sounds. For example, R2 stated: "This is the best option, because you can see where the sound is 


\begin{tabular}{lccc}
\hline & Floorplan & List & Waveform \\
\hline Understandability & $5.0(0.0)$ & $4.9(0.3)$ & $3.9(0.9)$ \\
Addresses needs & $4.8(0.4)$ & $4.3(0.6)$ & $2.4(1.3)$ \\
Likely to use in own & $4.5(0.9)$ & $4.2(1.0)$ & $2.1(1.2)$ \\
home & & & \\
\hline
\end{tabular}

Table 2: Mean (and SD) of participant responses to 5-point Likert-scale questions ( 5 is best), showing that the floorplan and list prototypes were preferred to the waveform prototype.

coming from and you know exactly where to look." Three participants valued its glanceability, in that they could notice the sound bubbles without paying active attention.

However, because this display always visualized sounds in the center of a room, all nine participants suggested finer-grained localization. "I want to see door knock on top of the door, not where it shows now [in the center of the lounge]" (R3). Other suggestions included using size or color to indicate loudness or pitch (4), or rings to indicate loudness, pitch (3) (see Figure 4A), and showing the time of occurrence (3). For example:

"Some deaf people may not know the difference between banging and tapping [on the door]. Having the blue [disk] change to red [to show loudness] would [solve] that." (R7)

The second most preferred design was the list prototype, which was R9's top choice and the remaining nine participants' second favorite. Participants liked that they could easily see a sound's exact timestamp (6) as well as a recorded history (5). Unlike the floorplan prototype where visualizations are transient, the list prototype records and displays sounds chronologically: "I am going to be busy doing other things and I don't have to check this out every 3 seconds" (R7). However, while the floorplan view was considered to be glanceable, five participants felt just the opposite about the list display, particularly when "there are many sounds in the list"(R9).

Finally, no participant selected the waveform prototype, finding that it required active visual attention (7), lacked information (4), and was hard to understand (3). However, seven participants saw the "benefit of using [waveform] for loudness information" (R2) and suggested combining it with other prototypes-by displaying a waveform in another column of the list prototype (Figure 4B), at the bottom of the floorplan prototype, or in the bubbles of the floorplan prototype. Similarly, six participants suggested a hybrid of the floorplan and list. Table 2 summarizes the questionnaire responses on the prototypes, which confirms that the waveform was not as well-received as the others.

Home-based Sound Awareness Themes
We now describe high-level themes that arose related to usability of a future in-home sound awareness system.

Actionability: All participants emphasized that a sound awareness system could help them perform desired tasks. Several suggestions for ensuring actionability arose, including: showing physical characteristics for sounds that require an action (8) (e.g., "[distinguish] loud banging from a soft door knock using loudness", R7), using vibrations on smartwatch or phone to notify about sounds (7), identifying that someone is calling for your attention as opposed to general conversation (7), and showing additional information for voices (e.g., tone) to identify if they demand attention (6). For example, R5 emphasized critical differences in sounds from her daughter's room:

"If my 9-year old daughter is sitting down in her room and talking normally, I am not concerned. If she's sobbing because she's in pain, then I need to know that."

For continuous sounds, because we only showed the beginning time, participants also wanted to know the end time to avoid needing to close the sound source (5):

"say a water flow... if it had a beginning and no end then that would be a problem. Perhaps have the circle stay on [in floorplan prototype] till the water stops flowing..."(R7)

Finally, three participants mentioned the importance of distinguishing real-life sounds from digital media like movies or music, as captured by R7: "I saw a 'smash' [on display]. I better go check if some-thing fell on ground but I don't know it's from TV."

Trust and confidence: Without trust, a sound awareness system is purposeless. We asked participants about three types of sensing errors that could arise: misattribution, false negatives, and false positives. Misattribution, that is, misrecognizing one sound for another, was seen as the most problematic, with most participants (7) feeling that these errors would undermine their trust in the system. However, false negatives, that is, not showing a sound that had occurred, would not pose a significant problem unless the sound was safety related (e.g., fire alarm) (7). For example,

"Whatever is more important and signifies a dangerous moment or something that is really life driven [should not be omitted]. I was at home alone and the carbon monoxide [alarm] was beeping for one week. My neighbor told me. That's when I corrected it. Those are important kinds of sounds I'd like to know. I don't mind if it [occasionally] misses out on sounds that are not important, because I've lived all my life like that." (R10) 
Finally, most participants felt that false positivesshowing a sound that did not occur-would be tolerable but annoying. For example,

"I am fine with it [...] But, if it repeatedly shows a sound coming from somewhere, and I keep on checking checking checking, we have to figure out what is going on, you know?" (R9)

After viewing the three options (location, list, waveform) for how to handle the system's uncertainty in identifying sounds, all participants said they would use the system even if it shows only a sound's location but not its identity (e.g., bathroom but not toilet flush); this finding reflects past work [14]. Eight participants also felt that the location plus other uncertain information could be useful, such as a high-level category (e.g., "an alarm-like sound", R8) or a list of possible sounds (e.g., "this sound could be a clock alarm or a microwave beep”, R4). As with the overall Wizard of $\mathrm{Oz}$ prototypes, the waveform was not wellreceived: eight participants felt that sound characteristics such as loudness would not be useful without location. Finally, only four participants wanted to see the confidence level (as shown in the list mockup) because it could reduce trust in the system and is not meaningful information. For example, R7 said: "If it said 52\% microwave beep or 23\% something else [...] you are still going to go and check which [sound] it is."

Finally, four participants wanted the system to provide an option to manually correct mistakes (e.g., to classify an unrecognized sound), for example, if a hearing friend noticed that the system was incorrect (R9).

Privacy: Privacy issues arose related to intimate sounds, activity tracking, and unwanted access to sound history. Toward the first issue, four participants felt uncomforatble about the system showing bathroom sounds. For example, "I don't want to know if someone is using toilet or what ever they are doing in the bathroom... It's their privacy, you know?" (R8). Conversely, six participants were more open to these sounds for accessibility reasons. For example, R1 emphasized the need for equal access to information: "Hearing people can hear all that, right? [So,] it's fair to have equal access to information. I want it all." There may be a cultural component to these preferences: the four participants who identify as hard of hearing were more concerned, but the five $\mathrm{D} /$ deaf participants and one deaf-blind participant were more open to these sounds.

As a second dimension of privacy, four participants noted that a sound awareness system could provide insight into other household members' or guests' activities-which may not be desired by either party. For example, "people [would] avoid coming to my house because they're been monitored each and every moment..." (R10). However, most participants (9) saw some value in tracking, for example, "I want to know what my cleaning lady's been doing. If she is using my computer without permission, then I can [know]" (R5), and "The value of this in monitoring a baby is significant" (R4). Consequently, to overcome privacy issues with activity tracking, some participants suggested including a setting in the system for selective sound recording (7) and letting other people know that their sounds are being recorded (5).

A final concern was household members or guests accessing the participant's past activities, particularly when a sound awareness display is installed in a shared space (6). For example, "I was thinking if this was installed in the living room, and some [guest] comes in, they can see what I've been doing?” (R8). Thus, two participants wanted the system to have a password option.

Information overload: A sound awareness system could lead to overload if many sounds occur simultaneously. Indeed, in the movie scenario, where participants viewed sounds while pretending to work on a laptop, they felt overwhelmed (9) and distracted (6). Thus, nine participants wanted the system to filter sounds and gave suggestions for doing so. For example, R7 wanted detailed customization:

"If I can enter [in the system] if [it shows] chair squeaking: okay I don't need to know that in future. Don't show that again... Also, after 5 days I can go back and select sounds from a list, like I don't need to hear this but I want to continue hearing this." (R7)

As in Study 1, five participants did not want to be aware of background noises unless it indicated a problem, such as the AC system clicking instead of humming (R5). Other suggestions to reduce overload included: limiting the system to mostly actionable sounds such as safetyrelated sounds (6) and human voices (5), and using colors to filter out sounds based on importance (5) (e.g., "red is fire alarm, blue is water [running]”, R3). Finally, four participants suggested using a manual "sensitivity" setting so the system can automatically filter sounds below a decibel level.

Contextualized feedback: Participants mentioned several contextual factors related to the home that affect sound awareness preferences: daily rhythms, domestic activities, and location in the home. In terms of daily rhythms, six participants wanted the system to limit sounds based on the time-for example, at night:

For night time you might want to [show] crying if the kids are in the other room. You wouldn't worry about siren outside or street noise or the air conditioning running. Those are daytime things. 


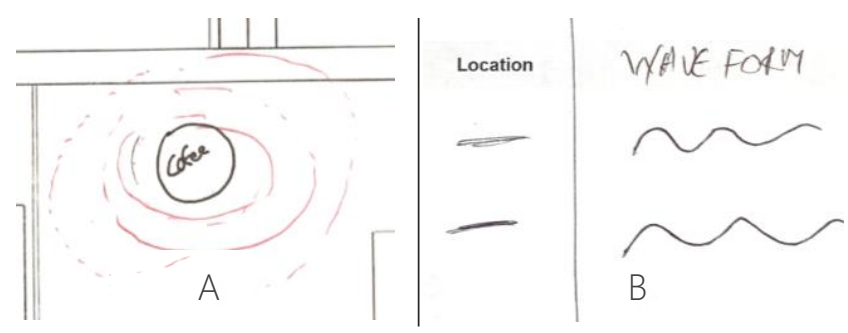

Figure 4: Design improvements sketched by participants. (A) R8 sketched rings around the sound bubbles of floorplan prototype to indicate loudness. (B) R10 added a column in the list design to show the sound waveform.

[...] Now, television. If it is happening between 10 o'clock at night and 5 in the morning [then I need to know]"(R5)

Four suggested adjusting the system based on domestic activities, including the user's current activity (e.g., only display urgent sounds when the user is working, R9) or the amount of activity in the home, such as: "Restrict to important sounds only when there's a large guest party, [because] I don't want to be distracted at that time" (R4).

Three participants wanted to adapt the system based on the user's current location. For example, R5 mentioned not wanting notifications for sounds when she was in a position to receive the same information visually (e.g., not needing a doorbell sound when she is in view of the front door). R2 mentioned not necessarily wanting to know about cooking sounds when he is cooking, but when "I am in bedroom and somebody is cooking, those sounds are important."

Other design considerations: Interestingly, participants were split on whether they wanted the system to show their own sounds. While five participants did not want to be aware about their own activities (e.g., "If I am [operating a faucet], I know that I am running water," R9), four participants felt they would benefit from knowing if they were making a loud noise or doing something incorrectly:

"I have had troubles when I talked loudly or played music loudly for other people. So, if [the system] could tell me the music is up too loud [that would help me respond]" (R5).

Finally, three participants suggested an option to train the system to identify their custom sounds, which supports work on personalized sound sensing [2]. For example,

"If I get a new microwave then the sound is different. I want to tell [the system], here's what my new microwave sounds like, [and] so, it can learn from it."(R1)

System installation: A future sound awareness system should seamlessly integrate into the home. Five participants emphasized the importance of accommodating existing home adaptations such as lightbased technologies and vibrating devices. For example, "The [vibrating] device under my bed could talk to the smartphone and vibrate if there's an emergency." (R3)

"I already have light [based tech] at my house. I want a coordinated system that [includes] these devices," (R10)

Some (5) wanted the system to integrate with other smart home systems. For example, R9 discussed using a sound sensing system alongside her security camera:

"I would look at [list prototype] first because I can scan it much more quickly than having to go through the security camera [recordings]. For instance, if I go through this list and see that the child cried for 30 minutes or something, then I can sync up with the video with the time stamp to see what went wrong." (R9)

Finally, participants were concerned about the cost and effort of the system installation and gave useful suggestions. All participants wanted to standardize the process of obtaining floorplan data to minimize overhead (e.g., by using templates). Six participants wanted to install the system in only the most-used rooms in the home to lower the cost. Finally, R4 and R2 suggested to show the "direction of the sound source instead of the specific location" (R4) if obtaining floorplan becomes difficult.

\section{Summary}

Participants appreciated the spatial layout and glanceability of the floorplan prototype, though also liked the list prototype; the waveform prototype was not wellreceived. We uncovered ways to increase the actionability of the system (e.g., showing both start and end times for continuous sounds) and to mitigate uncertainty of sound sensing (e.g., avoid misattribution errors, show location even without identity). Other themes arose, such as privacy related to other occupants or visitors, the need to consider contextual factors (daily rhythms, domestic activities, and location), and a desire for future systems to work with existing home adaptations and smart home technology.

\section{DISCUSSION}

These studies confirm past work with DHH participants on sounds of interest in the home [2,14], as well as identify new preferences for home-based sound awareness (e.g., integrate with existing infrastructure), and design considerations that manifest in unique ways in the home setting (e.g., privacy, the utility of displaying sounds in a home layout). While discussing our findings from the perspectives of domestic computing and accessibility 
research, below, we offer design recommendations and describe study limitations.

\section{Support for Domestic Processes}

Work in domestic computing supports that technologies should interweave into the living processes of the household [6,22]. Similarly, sound awareness technologies need to be contextually aware of home activities, and selectively display sounds. Several contextual parameters arose in our studies including the time of the day (e.g., night vs. day), user's current activity (e.g., working vs. idle), amount of activity in the home (e.g., a large guest party vs. quiet), and the user's location (e.g., kitchen vs. bedroom). Home-based sound awareness systems should be designed with these factors in mind, and further research with functional systems will help to understand how best to support these different contexts. Future technologies should also integrate with existing home adaptations of DHH users (e.g. vibratory wake-up alarms, R3) and other home systems (e.g., security camera, R9).

\section{A Shared Space}

The home is a complex and evolving space shared with other family members and guests, hence concerns of privacy arise [5]. Introduction of a sound awareness system into a space inhabited by deaf or hard of hearing occupants (which represents most of the households in our study) may change the notion of privacy-visual privacy means occluding line of sight, whereas sound can be sensed remotely. Investigating the implications of this difference will be important, particularly with consideration of cultural context. Indeed, our findings suggest that deaf/Deaf participants may have felt differently about intimate sounds than hard of hearing participants. Another dimension of privacy is about unwanted access to historical sound information. Placing the display out of view of the "public" areas of the house may mitigate this concern, but would also reduce the ease of accessing the information (some participants wanted displays in each room).

\section{Handing Uncertainty}

Home technologies need to gain the user's trust [6]. Similar to Mielke et al. [17], we found that false negatives (i.e., not showing an occurred sound) were deemed more tolerable than false positives (i.e., showing a sound that did not occur), and that misattribution (i.e., showing a wrong sound) would result in losing trust in the system. To supplement low confident detections, displaying additional cues such as location, possible list of sounds, or a general indication of the sound (e.g. "an alarm-like" sound) was found valuable (Matthew et al. [14] also found support for showing location). In contrast to Bragg et al. [2], however, our participants did not find value in displaying the confidence level. Future work should explore this in detail.

\section{Form Factor and UI Recommendations}

Among each prototype explored in Study 2, participants preferred the floorplan prototype the most because, as reflected in a past work [14], it visualizes sounds in a spatial layout. Our new list prototype, which displays a temporal history, was also well received. In contrast to the above past work [14], the waveform prototype was not preferred as a standalone design because of low information gain. Moreover, we did not expect that $\mathrm{DHH}$ people would want personal devices to help with sound awareness in the home rather than embedded wall displays, but we found a desire from some participants to complement the wall displays with alerts on a smartphone or a smartwatch (e.g., using "smartphone for quick feedback and walled devices for more in-depth information”, P3). Finally, though we did not conduct an evaluation, half of participants in Study 1 found promise in ambient displays (i.e., illuminating sound sources within a room), which should be further explored.

\section{A Note on Participant Diversity}

Our participants identified as Deaf, deaf or hard of hearing. Deaf (capital 'D') refers to people who belong to a Deaf culture with distinct norms and practices, whereas deaf (small 'd') and hard of hearing indicate people with hearing loss who may or may not identify with Deaf culture [4]. Further, the terms 'deaf' and 'hard of hearing' can refer to audiological differences in hearing level. Despite these differences, disability occurs on a spectrum and these groups have synergetic access needs and preferences (as echoed by the majority of our findings as well as past work, e.g., [2]). Recruiting a wide range of participants at this initial stage of research allowed us to explore solutions that would work for a diversity of users. Future work should also focus on the needs of specific subgroups within the $\mathrm{DHH}$ population.

\section{Limitations}

First, our findings are based on interviews, design probes and Wizard of $\mathrm{Oz}$ evaluation with a limited number of DHH participants. Future work should consider long term technology deployment in the home with a larger sample and compare perspectives. This will also help study the effects of technology on the family dynamics and behavior. Second, a controlled experiment to study distraction effects, and cognitive load would lead to more grounded results. Finally, most participants in our Study 2 (total six) lived alone and do not best represent a family 
household. In retrospect, we should have screened for this to better understand the social dynamics, and issues of sharing space.

\section{CONCLUSION}

This paper explored the domestic experiences of $\mathrm{DHH}$ people related to sounds, and preferences for future inhome sound awareness technology. Through semistructured formative interviews with $12 \mathrm{DHH}$ participants, Study 1 characterized perceptions of and experiences with the domestic sound, adaptations to deal with the sounds, and subjective reactions to initial sound awareness mockups. Informed by Study 1, we designed three sound awareness prototypes and conducted a Wizard-of-Oz evaluation with $10 \mathrm{DHH}$ participants in a home-like space (Study 2). Together, our findings related to actionability, personalization, contextual awareness, privacy, information overload and trust give direct guidance for future in-home sound awareness technology.

\section{ACKNOWLEDGMENTS}

We thank Sophie Tian, Liang He and Lisa Hutchinson. This work is supported by NSF Grant IIS-1763199, a Google Faculty Research Award, and a UW Reality Lab Grant.

\section{REFERENCES}

[1] Jeffrey P Bigham, Raja Kushalnagar, Ting-Hao Kenneth Huang, Juan Pablo Flores, and Saiph Savage. 2017. On How Deaf People Might Use Speech to Control Devices. In Proceedings of the 19th International ACM SIGACCESS Conference on Computers and Accessibility, 383-384.

[2] Danielle Bragg, Nicholas Huynh, and Richard E. Ladner. 2016. A Personalizable Mobile Sound Detector App Design for Deaf and Hard-of-Hearing Users. In Proceedings of the 18th International ACM SIGACCESS Conference on Computers and Accessibility - ASSETS '16, 3-13.

[3] Virginia Braun and Victoria Clarke. 2006. Using thematic analysis in psychology. Qualitative Research in Psychology 3, 2: 77-101.

[4] Anna Cavender and Richard E Ladner. 2008. Hearing impairments. In Web accessibility. Springer, 25-35.

[5] Eun Kyoung Choe, Sunny Consolvo, Jaeyeon Jung, Beverly Harrison, and Julie A Kientz. 2011. Living in a glass house: a survey of private moments in the home. In Proceedings of the 13th international conference on Ubiquitous computing, 41-44.

[6] Audrey Desjardins, Ron Wakkary, and William Odom. 2015. Investigating genres and perspectives in $\mathrm{HCI}$ research on the home. In Proceedings of the 33rd Annual ACM Conference on Human Factors in Computing Systems, 3073-3082.

[7] Mari Carmen Domingo. 2012. An overview of the Internet of Things for people with disabilities. Journal of Network and Computer Applications 35, 2: 584-596.

[8] Leah Findlater, Bonnie Chinh, Dhruv Jain, Jon Froehlich, Raja Kushalnagar, and Angela Carey Lin. 2019. Deaf and Hard-ofhearing Individuals' Preferences for Wearable and Mobile Sound Awareness Technologies. In SIGCHI Conference on Human Factors in
Computing Systems (CHI).

[9] Raymond Fok, Harmanpreet Kaur, Skanda Palani, Martez E Mott, and Walter S Lasecki. 2018. Towards More Robust Speech Interactions for Deaf and Hard of Hearing Users.

[10] Jon Froehlich, Leah Findlater, Marilyn Ostergren, Solai Ramanathan, Josh Peterson, Inness Wragg, Eric Larson, Fabia Fu, Mazhengmin Bai, Shwetak Patel, and others. 2012. The design and evaluation of prototype eco-feedback displays for fixture-level water usage data. In Proceedings of the SIGCHI conference on human factors in computing systems, 2367-2376.

[11] Dhruv Jain, Leah Findlater, Christian Volger, Dmitry Zotkin, Ramani Duraiswami, and Jon Froehlich. 2015. Head-Mounted Display Visualizations to Support Sound Awareness for the Deaf and Hard of Hearing. In Proceedings of the 33rd Annual ACM Conference on Human Factors in Computing Systems, 241--250.

[12] Sergei Kochkin. 2000. MarkeTrak V:"Why my hearing aids are in the drawer" The consumers' perspective. The Hearing fournal 53, 2: 34-36.

[13] Tara Matthews. 2006. Designing and Evaluating Glanceable Peripheral Displays. In Proceedings of the 6th Conference on Designing Interactive Systems (DIS ’06), 343-345.

[14] Tara Matthews, Janette Fong, F. Wai-Ling Ho-Ching, and Jennifer Mankoff. 2006. Evaluating non-speech sound visualizations for the deaf. Behaviour \& Information Technology 25, 4: 333-351.

[15] Abby McCormack and Heather Fortnum. 2013. Why do people fitted with hearing aids not wear them? International fournal of Audiology 52, 5: 360-368.

[16] Matthias Mielke and Rainer Brück. 2015. A Pilot Study about the Smartwatch as Assistive Device for Deaf People. In Proceedings of the 17th International ACM SIGACCESS Conference on Computers \& Accessibility, 301-302.

[17] Matthias Mielke and Rainer Brueck. 2015. Design and evaluation of a smartphone application for non-speech sound awareness for people with hearing loss. In Engineering in Medicine and Biology Society (EMBC), 2015 37th Annual International Conference of the IEEE, 5008-5011.

[18] James Pierce, Diane J Schiano, and Eric Paulos. 2010. Home, habits, and energy: examining domestic interactions and energy consumption. In Proceedings of the SIGCHI Conference on Human Factors in Computing Systems, 1985-1994.

[19] Alisha Pradhan, Kanika Mehta, and Leah Findlater. 2018. Accessibility Came by Accident: Use of Voice-Controlled Intelligent Personal Assistants by People with Disabilities. In Proceedings of the 2018 CHI Conference on Human Factors in Computing Systems, 459.

[20] Liu Sicong, Zhou Zimu, Du Junzhao, Shangguan Longfei, Jun Han, and Xin Wang. 2017. UbiEar: Bringing Location-independent Sound Awareness to the Hard-of-hearing People with Smartphones. Proceedings of the ACM on Interactive, Mobile, Wearable and Ubiquitous Technologies 1, 2: 17.

[21] Daisuke Uriu and William Odom. 2016. Designing for domestic memorialization and remembrance: a field study of fenestra in Japan. In Proceedings of the 2016 CHI Conference on Human Factors in Computing Systems, 5945-5957.

[22] Allison Woodruff, Sally Augustin, and Brooke Foucault. 2007. Sabbath day home automation: it's like mixing technology and religion. In Proceedings of the SIGCHI conference on Human factors in computing systems, 527-536.

[23] Rayoung Yang and Mark W Newman. 2013. Learning from a learning thermostat: lessons for intelligent systems for the home. In Proceedings of the 2013 ACM international joint conference on Pervasive and ubiquitous computing, 93-102.

[24] DeafSpace - $\quad$ Gallaudet University. https://www.gallaudet.edu/campus-design-and-planning/deafspace

[25] Amazon Echo Show. https://www.digitaltrends.com/smart-homereviews/amazon-echo-show-review/ 